Ciência Florestal, Santa Maria, v. 22, n. 4, p. 821-831, out.-dez., 2012

ISSN 0103-9954

\title{
BALANÇO NUTRICIONAL, PRODUÇÃO DE ÓLEO ESSENCIAL E MADEIRA DE Corymbia citriodora Hill \& Johnson COM APLICAÇÃO DE LODO DE ESGOTO E FERTILIZANTE MINERAL
}

\author{
NUTRITIONAL BALANCE, ESSENTIAL OIL AND WOOD PRODUCTION FROM Corymbia \\ citriodora Hill \& Johnson WITH SEWAGE SLUDGE AND MINERAL FERTILIZER APPLICATIONS
}

\author{
Paulo Henrique Müller da Silva ${ }^{1}$ Fabio Poggiani ${ }^{2}$ Alexandre de Vicente Ferraz ${ }^{3}$ \\ Ricardo Michael de Melo Sixel ${ }^{4}$ José Otavio Brito ${ }^{5}$
}

\begin{abstract}
RESUMO
O Corymbia citriodora é uma das espécies florestais mais difundidas no Brasil, sendo um dos motivos a diversidade de uso da espécie, pois produz madeira de qualidade e as folhas podem ser utilizadas para produção de óleo essencial. Entretanto, é uma espécie que não tem muitos estudos relacionados ao efeito do manejo no balanço nutricional. O objetivo deste trabalho foi avaliar a produção de biomassa e o balanço nutricional no sistema convencional de produção de óleo essencial e madeira de Corymbia citriodora com a aplicação de lodo de esgoto. O delineamento experimental utilizado foi em blocos casualizados, com quatro repetições e dois tratamentos, sendo eles: 1 - Fertilização com 10 tha $^{-1}$ (base seca) de lodo de esgoto, complementado com $\mathrm{K}+\mathrm{B}$, e 2 - Fertilização mineral. Foi avaliada a produção de biomassa aérea das árvores e dos respectivos componentes (folha, galho, casca e lenho), a exportação de nutrientes via colheita da folhas, a produção de óleo essencial e da madeira aos quatro anos de idade. Observou-se que as árvores fertilizadas com lodo produziram $20 \%$ mais folhas durante o ciclo de produção, o que resultou em maior produção de óleo essencial. Além disso, o tratamento com a aplicação do lodo produziu $14,2 \mathrm{t} \mathrm{ha}^{-1}$ ano $^{-1}$ de biomassa lenhosa, que foi $27 \%$ superior ao tratamento com fertilização mineral. Para ambos os tratamentos o balanço do $\mathrm{N}$ foi negativo, sendo que a exportação de $\mathrm{N}$ no tratamento com aplicação de lodo de esgoto (-45 kg ha $\left.{ }^{-1}\right)$ foi 4 vezes menor que a observada no tratamento com fertilização mineral (-185 kg ha $\left.{ }^{-1}\right)$. Com o presente estudo pode-se concluir que a aplicação de lodo de esgoto em plantios de Corymbia citriodora beneficia a produção de biomassa foliar, óleo essencial e madeira, além de propiciar melhor balanço nutricional.
\end{abstract}

Palavras-chave: Eucalyptus citriodora; biossólido; exportação de nutrientes.

\section{ABSTRACT}

The Corymbia citriodora is one of the most important forest species in Brazil and the reason is the diversity of its use, because it produces good quality wood and the leaves may be used for essential oil production. Although, there are not many studies about species and the handling effect in the nutritional balance. This study aimed to evaluate the biomass production and nutrient balance in the conventional production of

1. Engenheiro Florestal, Msc., Doutorando do Programa de Recursos Florestais na Escola Superior Luiz de Queiróz, Universidade de São Paulo, Assistente Técnico-Científico do Instituto de Pesquisas e Estudos Florestais, Av. Pádua Dias, 11, Caixa Postal 530, CEP 13400-970, Piracicaba (SP).paulohenrique@usp.br

2. Biólogo, Dr., Professor Titular do Departamento de Ciências Florestais, Escola Superior Luiz de Queiróz, Universidade de São Paulo, Av. Pádua Dias, 11, Caixa Postal 09, CEP 13418-900, Piracicaba (SP). fpoggian@esalq.usp.br

3. Engenheiro Florestal, Msc., Doutorando do Programa de Recursos Florestais, Escola Superior Luiz de Queiróz, Universidade de São Paulo, Av. Pádua Dias, 11, Caixa Postal 09, CEP 13418-900, Piracicaba (SP). avferraz@esalq.usp.br

4. Engenheiro Florestal, Mestrando do Programa de Recursos Florestais, Escola Superior Luiz de Queiróz, Universidade de São Paulo, Av. Pádua Dias, 11, Caixa Postal 09, CEP 13418-900, Piracicaba (SP). sixelr@gmail.com

5. Engenheiro Florestal, Dr, Professor Titular do Departamento de Ciências Florestais, Escola Superior Luiz de Queiróz, Universidade de São Paulo, Av. Pádua Dias, 11, Caixa Postal 09, CEP 13418-900, Piracicaba (SP). jobrito@usp.br

Recebido para publicação em 16/11/2009 e aceito em 16/08/2011 
essential oil and wood of Corymbia citriodora with sewage sludge application. The experiment design established was the randomized blocks, with four replicates and two treatments: 1 - fertilization with 10 tons $\mathrm{ha}^{-1}$ (dry mass) of sewage sludge, supplemented with $\mathrm{K}$ and $\mathrm{B}$, and 2 - mineral fertilization. It was evaluated the aerial biomass production, the nutrient export of the leaves, the essential oil and wood production at four years old. The trees that received application of sewage sludge produced $20 \%$ more leaves biomass than the trees with mineral fertilization, resulting in larger oil production. Besides, the trees with sewage sludge application produced 14.2 tons $\mathrm{ha}^{-1} \mathrm{yr}^{-1}$ of woody biomass that was $27 \%$ higher than the treatment with mineral fertilization. For both treatments the $\mathrm{N}$ balance was negative, but treatment with sewage sludge application (-45 $\left.\mathrm{kg} \mathrm{ha}^{-1}\right)$ was four times lower than the observed on mineral fertilization treatment $\left(-185 \mathrm{~kg} \mathrm{ha}^{-1}\right)$. It may be concluded in this paper that the application of sewage sludge benefits the production of leaves biomass, essential oil and wood, besides result better nutritional balance of the Corymbia citriodora production system.

Keywords: Eucalyptus citriodora; bio-solids; export of nutrients.

\section{INTRODUÇÃO}

O Corymbia citriodora, mais conhecido como Eucalyptus citriodora, tem papel de destaque no setor florestal brasileiro, pois apresenta grande diversidade de usos, devido às características da madeira e pela produção de óleo essencial, cujo principal constituinte é o citronelal (BATISH et al., 2006). O óleo essencial é utilizado in natura para a aromatização de ambientes ou na fabricação de desinfetantes, detergentes, sabões, mascarantes industriais e perfumaria.

As plantações de eucalipto para a produção de óleo geralmente são mais adensadas, com aproximadamente 3300 árvores por hectare, e para obtenção das folhas para destilação são realizadas colheitas anuais que retiram de maneira manual em torno de 2/3 da copa. Aos 4 ou 5 anos de idade é realizado o corte das árvores que serão conduzidas por talhadia. O corte é necessário, pois a altura das árvores dificulta a colheita, sendo obtidas as folhas para destilação e a madeira normalmente é utilizada para fins energéticos (lenha e carvão) ou para produção de mourões. É um sistema intensivo de produção se comparado com outros sistemas utilizados pelas empresas florestais ligadas a outros ramos, cuja matéria-prima tem ciclos de corte mais longos (6 ou 7 anos) sem a extração das folhas.

Não existem muitos estudos relacionados ao manejo ou à sustentabilidade do sistema de produção de óleo e madeira do Corymbia citriodora, sendo que um dos pontos importantes na busca da sustentabilidade do ecossistema florestal é a reposição dos nutrientes exportados com a colheita. As folhas, que são coletadas para a extração do óleo, apresentam elevadas concentrações dos nutrientes em relação aos demais componentes das árvores (PEREIRA et al., 1984; POGGIANI et al., 1984).

A utilização do lodo de esgoto é uma opção para repor os nutrientes exportados com a colheita das folhas e da madeira, pois o lodo além de possuir elevados teores de nitrogênio, fósforo e micronutrientes, apresenta liberação mais lenta dos nutrientes para o solo em relação ao fertilizante mineral, propiciando uma melhor sincronia entre a disponibilização dos nutrientes e sua absorção pelas raízes (POGGIANI et al., 2006). O lodo de esgoto, quando devidamente tratado, pode ser utilizado como fertilizante orgânico, visando à reposição de nutrientes ao solo em plantações de eucaliptos (HARRISON et al., 2003). Em 29 de agosto de 2006, o Conselho Nacional do Meio Ambiente (CONAMA, 2006) criou a Resolução $n^{\circ}$ 375 , regulamentando a utilização agrícola do lodo de esgoto ao definir critérios e medidas quanto à qualidade e à forma de aplicação do lodo ao solo. Em São Paulo a norma P-4230 da CETESB (1999) estabelece os limites e a taxa máxima de aplicação anual de metais em solos tratados com lodo de esgoto e a carga máxima acumulada pela aplicação.

O objetivo deste trabalho foi avaliar a produção de biomassa lenhosa, foliar e o balanço nutricional em um ciclo de produção convencional visando à produção do óleo essencial e da madeira de Corymbia citriodora fertilizado com lodo de esgoto em comparação a fertilização mineral.

\section{MATERIAL E MÉTODO}

\section{Descrição da área de estudo}

$\mathrm{O}$ experimento foi instalado em fevereiro de 2005, na Estação Experimental de Ciências 
Florestais de Itatinga, vinculada ao Departamento de Ciências Florestais da Escola Superior de Agricultura "Luiz de Queiroz", localizada no município de Itatinga - SP, na latitude $23^{\circ} 02^{\prime}$ e longitude 48³7', com altitude média de $830 \mathrm{~m}$. O clima do município de Itatinga, segundo classificação de Köppen, é do tipo Cwa - mesotérmico úmido com inverno seco e precipitação média mensal de $1300 \mathrm{~mm}$. A temperatura média anual é de $19,4^{\circ} \mathrm{C}$, sendo a mínima de $16,3{ }^{\circ} \mathrm{C}$ no mês de julho e a máxima de $21,9{ }^{\circ} \mathrm{C}$ no mês de janeiro (ROLIM e SENTELHAS, 1999). O solo da área experimental é um Latossolo Vermelho-Amarelo com textura médio-arenosa (EMBRAPA, 2006), de relevo plano e baixa fertilidade natural (Tabela 1). Esse tipo de solo era primitivamente coberto por vegetação de cerrado, sendo representativo das áreas onde se pratica atualmente a silvicultura do eucalipto no estado de São Paulo.

\section{Delineamento experimental}

$\mathrm{O}$ delineamento experimental utilizado foi em blocos casualizados, com 4 repetições e dois tratamentos, sendo eles: 1 - Fertilização com $10 \mathrm{t} \mathrm{ha}^{-1}$ (base seca) de lodo esgoto, complementado com $80 \mathrm{~kg} \mathrm{ha}^{-1}$ de Ke $3 \mathrm{~kg} \mathrm{ha}^{-1}$ de B (LE) e 2 Fertilização mineral N-P-K (convencional) com $115 \mathrm{~kg} \mathrm{ha}^{-1}$ de $\mathrm{N}+60 \mathrm{~kg} \mathrm{ha}^{-1}$ de $\mathrm{P}+80 \mathrm{~kg} \mathrm{ha}^{-1}$ de $\mathrm{K}+440 \mathrm{~kg} \mathrm{ha}{ }^{-1}$ de $\mathrm{Ca}+160 \mathrm{~kg} \mathrm{ha}^{-1} \mathrm{de} \mathrm{Mg}+$
$5 \mathrm{~kg} \mathrm{ha}^{-1}$ de S e $3 \mathrm{~kg} \mathrm{ha}^{-1}$ de B (FM). Cada parcela foi ocupada por 100 árvores, implantadas no espaçamento de $3 \times 1$ metro, totalizando uma área $300 \mathrm{~m}^{2}$. Todavia, foi estabelecida em cada parcela uma faixa de bordadura dupla, o que proporcionou uma área útil de $108 \mathrm{~m}^{2}$ com 36 árvores, que foram efetivamente avaliadas.

O lodo de esgoto foi aplicado manualmente no solo, em dose única, logo após o plantio, em pequenas porções com o auxílio de um balde, numa faixa com aproximadamente $0,3 \mathrm{~m}$ de largura sobre a linha de plantio dos eucaliptos. A complementação com potássio e boro via aplicação respectivamente de $\mathrm{KCl}$ e Borax ${ }^{\circledR}$, foi necessária no tratamento LE devido aos baixos teores de $\mathrm{K}$ e $\mathrm{B}$ no lodo gerado pela ETE de Barueri-SP (Tabela 2). A forma e os períodos de aplicação do $\mathrm{KCl}$ e do Borax utilizados no tratamento LE, bem como dos fertilizantes minerais no tratamento $\mathrm{FM}$, foram realizados de acordo com as recomendações do Boletim Técnico 100 (RAIJ et al., 1996).

A dose aplicada de 10 toneladas de lodo por hectare foi determinada a partir de resultados previamente obtidos na estação Experimental de Ciências Florestais de Itatinga e foi baseada em critérios técnicos, silviculturais e ecológicos, visando aumentar a produtividade, sem causar efeitos indesejáveis no ambiente (POGGIANI et al., 2006; SILVA et al., 2008).

TABELA 1: Atributos químicos ${ }^{\mathrm{A}} \mathrm{e}$ físicos ${ }^{\mathrm{B}}$ do solo antes da instalação do experimento.

TABLE 1: $\quad$ Chemical $^{\mathrm{A}}$ and Physical ${ }^{\mathrm{B}}$ attributes of the soil before of the installation of experiment.

\begin{tabular}{|c|c|c|c|c|c|c|c|c|c|c|}
\hline Prof. & $\mathrm{pH}$ & M.O & $P_{\text {resina }}$ & K & $\mathrm{Ca}$ & $\mathrm{Mg}$ & $\mathrm{H}+\mathrm{Al}$ & SB & CTC & $\mathrm{V}$ \\
\hline$(\mathrm{cm})$ & $\mathrm{CaCl}_{2}$ & $\mathrm{~g} \mathrm{dm}^{-3}$ & $m g \mathrm{dm}^{-3}$ & \multicolumn{6}{|c|}{ 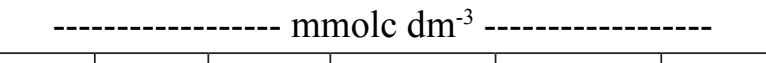 } & $\%$ \\
\hline $0-5$ & 4,0 & 26 & 9 & 0,7 & 5 & 4 & 71 & 9,7 & 81 & 12 \\
\hline $5-10$ & 4,0 & 17 & 6 & 0,6 & 3 & 2 & 57 & 5,6 & 63 & 8,9 \\
\hline $10-20$ & 4,0 & 13 & 7 & 0,6 & 2 & 2 & 44 & 4,6 & 49 & 9,5 \\
\hline Prof. & B & $\mathrm{Cu}$ & $\mathrm{Fe}$ & $\mathrm{Mn}$ & $\mathrm{Zn}$ & \multirow{2}{*}{\multicolumn{2}{|c|}{ Areia total }} & Silte & \multicolumn{2}{|c|}{ Argila total } \\
\hline$(\mathrm{cm})$ & \multicolumn{5}{|c|}{ - } & & & $\%$ & & \\
\hline $0-5$ & 0,25 & 0,5 & 104 & 2,5 & 0,8 & & 81 & 4,0 & \multicolumn{2}{|c|}{15,0} \\
\hline $5-10$ & 0,21 & 0,6 & 76 & 1,1 & 0,4 & & 78 & 4,0 & \multicolumn{2}{|c|}{18,0} \\
\hline $10-20$ & 0,19 & 0,7 & 55 & 0,6 & 0,3 & & 78 & 4,0 & \multicolumn{2}{|c|}{18,0} \\
\hline
\end{tabular}

Em que: ${ }^{\mathrm{A}}=$ Análise química realizada segundo metodologia apresentada por Raij et al. (1987); ${ }^{\mathrm{B}}=$ Análise física realizada segundo metodologia proposta por Camargo et al. (1986). 
TABELA 2: Características físicas e químicas do lodo de esgoto produzido pela estação de tratamento de esgoto de Barueri (SABESP-SP).

TABLE 2: Physical and chemical characteristics of sewage sludge produced by the wastewater treatment plant of Barueri (SABESP-SP).

\begin{tabular}{|c|c|}
\hline Determinações $^{\mathrm{a}}$ & Quantidade \\
\hline $\mathrm{pH}$ em $\mathrm{CaCl}_{2} 0,01 \mathrm{~mol} \mathrm{~L}^{-1}$ & 7,3 \\
\hline Densidade & $1,03 \mathrm{~g} \mathrm{~cm}^{-3}$ \\
\hline Umidade $^{\mathrm{b}}$ & $77,7 \%$ \\
\hline Matéria Orgânica (combustão) ${ }^{\mathrm{b}}$ & $54,6 \%$ \\
\hline Carbono (orgânico e mineral) & $30,8 \%$ \\
\hline Nitrogênio $(\mathrm{N})$ & $32,7 \mathrm{~g} \mathrm{~kg}^{-1}$ \\
\hline Fósforo $\left(\mathrm{P}_{2} \mathrm{O}_{5}\right)$ & $32,7 \mathrm{~g} \mathrm{~kg}^{-1}$ \\
\hline Potássio $\left(\mathrm{K}_{2} \mathrm{O}\right)$ & $2,7 \mathrm{~g} \mathrm{~kg}^{-1}$ \\
\hline Cálcio (Ca) & $25 \mathrm{~g} \mathrm{~kg}^{-1}$ \\
\hline Magnésio (Mg) & $4,9 \mathrm{~g} \mathrm{~kg}^{-1}$ \\
\hline Enxofre (S) & $6,6 \mathrm{~g} \mathrm{~kg}^{-1}$ \\
\hline Cobre $(\mathrm{Cu})$ & $570 \mathrm{mg} \mathrm{kg}^{-1}$ \\
\hline Manganês (Mn) & $194 \mathrm{mg} \mathrm{kg}^{-1}$ \\
\hline Zinco (Zn) & $2,3 \mathrm{~g} \mathrm{~kg}^{-1}$ \\
\hline Ferro $(\mathrm{Fe})$ & $39 \mathrm{~g} \mathrm{~kg}^{-1}$ \\
\hline Boro (B) & $9 \mathrm{mg} \mathrm{kg}^{-1}$ \\
\hline
\end{tabular}

Em que: ${ }^{\mathrm{a}}=$ Valores obtidos com base na matéria seca; ${ }^{\mathrm{b}}=$ Valores em porcentagem referem-se à relação massa/massa.

\section{Coleta e análise dos dados}

A produção de biomassa foliar para extração do óleo essencial foi determinada anualmente (no mês de janeiro) até o $4^{\circ}$ ano do ciclo de produção. Para isso, do $1^{\circ}$ ao $3^{\circ}$ ano de cultivo dos eucaliptos, foram efetuadas desramas de todas as árvores das parcelas, sendo retirados $2 / 3$ dos galhos + folhas pertencentes à região inferior das copas. Em cada coleta eram amostradas 6 árvores por parcela (24 árvores por tratamento) para realização das análises laboratoriais.

No $4^{\circ}$ ano, além da biomassa de galhos e folhas, foi quantificada também a biomassa de casca e lenho bem como a exportação de nutrientes, simulando o final do ciclo de produção com a colheita total das folhas e da madeira.

Depois do abate e do desmembramento da parte aérea das árvores, cada componente foi pesado em balança digital, em seguida, foram coletadas amostras para a determinação da umidade e da concentração dos nutrientes em laboratório. Além disso, as amostras de folha e tronco foram utilizadas respectivamente para a determinação da concentração de óleo essencial e da percentagem de casca e lenho.

As amostras foram secas em estufa com ventilação forçada a $60{ }^{\circ} \mathrm{C}$ e pesadas em balança digital com precisão de 0,01 g. Após a secagem e a determinação da umidade, as subamostras foram moídas em moinho tipo Willey e encaminhadas ao Laboratório de Ecologia Aplicada da ESALQ/ USP para análise da concentração dos macro e micronutrientes, de acordo Malavolta et al. (1997). A biomassa seca das folhas, lenho, galho e casca foi estimada a partir da biomassa verde pesada em campo e da umidade determinada nas subamostras coletadas. A exportação de nutrientes com a colheita das árvores foi estimada a partir da biomassa seca dos componentes da parte aérea das árvores multiplicada pelas respectivas concentrações de nutrientes.

Para estimar a produção de óleo, logo após a colheita as amostras foram destiladas por imersão em água, pelo período de 1 hora. A hidrodestilação do óleo essencial foi realizada em um destilador tipo Clevenger MA553, com volume nominal de 2 litros.

Os resultados foram inicialmente analisados quanto às pressuposições estatísticas básicas e a necessidade de transformação dos dados. Após esta etapa, os resultados foram submetidos à análise de variância e as médias foram comparadas pelo teste $\mathrm{t}$ de Student, com nível de significância de $5 \%$. O software estatístico utilizado foi o SAS 9.1 for 
Windows (SAS INSTITUTE, 2002-2003).

\section{RESULTADOS E DISCUSSÃO}

A produção de biomassa foliar do $1^{\circ}$ ao $3^{\circ}$ ano do ciclo de cultivo, bem como a produção de óleo essencial, não diferiu entre os tratamentos com lodo de esgoto e com fertilizante mineral (Tabela 3). Em média, a produção total de biomassa foliar e de óleo essencial nesses três primeiros anos foi de, respectivamente, $11,4 \mathrm{t} \mathrm{ha}^{-1}$ e $192,5 \mathrm{~L} \mathrm{ha}^{-1}$.

Por outro lado, a biomassa foliar e o volume de óleo essencial produzidos no final do $4^{\circ}$ ano do ciclo de cultivo, com o corte raso das árvores, foram cerca de $30 \%$ superiores no tratamento com lodo de esgoto comparado à fertilização mineral. Somados os períodos de colheita do $1^{\circ}$ ao $4^{\circ}$ ano do ciclo de produção, constatou-se que a fertilização com o lodo proporcionou maior biomassa foliar e, consequentemente, maior volume de óleo essencial (em torno de $14 \%$ ) com relação ao tratamento com fertilizante mineral. Segundo estudos conduzidos por Silva et al. (2009), a aplicação de lodo de esgoto em plantações de Corymbia citriodora não altera a qualidade do óleo essencial produzido.

$\mathrm{Na}$ avaliação realizada aos 4 anos, a biomassa de lenho, casca, galhos e folhas produzidas no tratamento com lodo de esgoto foi de 25 a $30 \%$ superior ao tratamento com fertilizante mineral(Figura 1). A biomassa aérea total produzida no tratamento com lodo de esgoto foi aproximadamente de $75 \mathrm{t} \mathrm{ha}^{-1}$ e a biomassa aérea total produzida no tratamento com fertilizante mineral foi cerca de $60 \mathrm{t} \mathrm{ha}^{-1}$, ou seja, $20 \%$ inferior. O incremento médio anual (IMA) de biomassa lenhosa no tratamento com fertilizante mineral foi de $11,2 \mathrm{t} \mathrm{ha}^{-1}$, inferior ao tratamento com lodo de esgoto, cujo IMA foi de 14,2 $\mathrm{t} \mathrm{ha}^{-1}$.

Em ambos os tratamentos, o lenho correspondeu a $76 \%$ da biomassa aérea total, a casca $10 \%$, as folhas $7,5 \%$ e os galhos $6,5 \%$. De forma semelhante, Andrade et al. (2006) ao estudarem a produção de biomassa em plantio de Eucalyptus grandis fertilizado com lixo urbano, verificaram que o lenho que é retirado do sítio no momento da colheita corresponde, em média, a $80 \%$ da biomassa aérea total, restando $20 \%$ dos outros compartimentos que podem ser mantidos na área, após a exploração. Comparada ao estudo de Andrade et al. (2006), observou-se que a porcentagem de casca $(13,5 \%)$ em relação à biomassa do tronco de Corymbia citriodora foi superior ao observado para o Eucalyptus grandis (9 \%), tanto no tratamento com lodo quanto no tratamento com fertilizante mineral.

Deve-se ressaltar que as quantidades de nutrientes adicionados ao solo nos tratamentos com a aplicação de lodo de esgoto e com o fertilizante mineral foram diferentes, entretanto, deve-se considerar também que o lodo decompõe-se lentamente, disponibilizando de forma gradual os nutrientes ao longo do tempo. Segundo Laclau et al. (2004) o sistema radicular dos eucaliptos possibilita a absorção dos nutrientes liberados pelo lodo de esgoto ao longo do ano, independente da estação climática.

A absorção dos nutrientes fornecidos pelo lodo resultou em maior incremento da biomassa aérea total produzida pelos eucaliptos, comparado ao incremento obtido no tratamento com fertilizante mineral. Contudo, não foram observadas diferenças significativas nas concentrações dos macronutrientes e micronutrientes em nenhum dos componentes da parte aérea das árvores, aos 4 anos de idade (Tabelas 4 e 5).

TABELA 3: Biomassa foliar total (base seca) e volume total de óleo essencial produzidos em dois períodos (do $1^{\circ}$ ao $3^{\circ}$ e no $4^{\circ}$ ano) do ciclo de cultivo, nos tratamentos com lodo de esgoto (LE) e com fertilizante mineral (FM).

TABLE 3: Leaves total biomass (dry weight) and total volume of essential oil produced in two periods (from 1 st to 3 rd and 4 th year) of the cultivation cycle in treated sewage sludge (LE) and mineral fertilizer (FM).

\begin{tabular}{cccccccc}
\hline \multirow{2}{*}{ Período de colheita } & \multicolumn{3}{c}{ Biomassa Foliar $\left(\mathrm{t} \mathrm{ha}^{-1}\right)$} & \multicolumn{3}{c}{ Óleo essencial $\left(\mathrm{L} \mathrm{ha}^{-1}\right)$} \\
& LE & \multicolumn{2}{c}{ FM } & LE & FM \\
\hline $1^{\circ}$ ao $3^{\circ}$ ano $(2 / 3$ da copa $)$ & 11,9 & 10,9 & ns & 200 & 185 & $\mathrm{~ns}$ \\
$4^{\circ}$ ano $(100 \%$ da copa $)$ & 5,6 & 4,4 & $*$ & 92 & 72 & $*$ \\
Total & 17,5 & 15,3 & $*$ & 292 & 257 & $*$ \\
\hline
\end{tabular}

Em que: * = Diferença significativa a $5 \%$ de probabilidade pelo teste $\mathrm{t}$. ${ }^{\text {ns }}$ Diferença não significativa pelo teste $\mathrm{t}$ $(\mathrm{P}>0,05)$. 


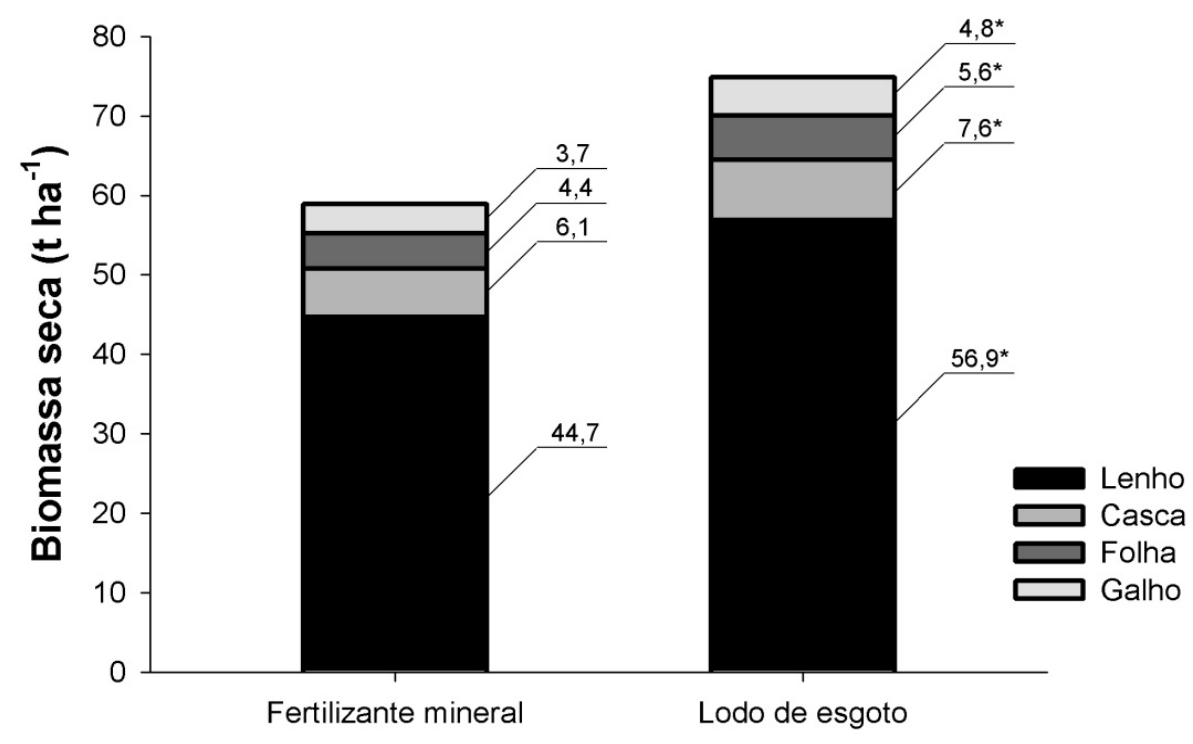

\section{Tratamento}

FIGURA 1: Biomassa produzida nos diferentes componentes da parte aérea do Corymbia citriodora, aos 4 anos de idade, fertilizado com lodo de esgoto e com fertilizante mineral NPK. Para um mesmo componente, a presença do asterisco $(*)$ significa diferença estatística a $5 \%$ de probabilidade pelo teste t.

FIGURE 1: Shoot biomass produced in different compartments of Corymbia citriodora, 4 years old, fertilized with sewage sludge and mineral fertilizer. For the same component, the presence of the asterisk $(*)$ denotes statistical difference at $5 \%$ probability by $t$ test.

As concentrações foliares de nitrogênio (Tabela 4) foram similares à concentração de $\mathrm{N}$ observadas por Zaia e Gama-Rodrigues (2004), em folhas de Eucalyptus grandis e Eucalyptus camaldulensis (em média 16,1 $\mathrm{g} \mathrm{kg}^{-1}$ ) aos seis anos de idade. Estes autores, ao estudarem a ciclagem e o balanço de nutrientes em plantios de eucaliptos na região norte fluminense, verificaram também que a concentração foliar de $\mathrm{N}$ para o Eucalyptus pellita foi significativamente inferior $\left(12,7 \mathrm{~g} \mathrm{~kg}^{-1}\right)$ às demais espécies de eucaliptos estudadas. Tendo este fato em vista, Santana et al. (1999) comentam que a variação nas quantidades de nutrientes exportados por diferentes materiais genéticos pode ser resultante tanto da biomassa produzida quanto da capacidade desses materiais para absorção, distribuição e utilização de nutrientes.

Comparados os componentes aéreos da árvore (Tabelas 4 e 5), as maiores concentrações de $\mathrm{N}, \mathrm{P}, \mathrm{K}, \mathrm{Mg}, \mathrm{S}, \mathrm{B}$ e $\mathrm{Cu}$ foram verificadas nas folhas, e de Ca na casca; e as menores concentrações desses nutrientes foram constatadas no lenho do Corymbia citriodora. Segundo Bellote e Silva (2005), cada um dos componentes da árvore possui concentração de nutrientes minerais relacionada com sua funções fisiológicas e morfológicas, havendo um gradiente que geralmente apresenta a seguinte sequência de concentração: folha $>$ casca $>$ ramo $>$ lenho (alburno+cerne).

Na tabela 5, destaca-se que a concentração de Zinco e Cobre na biomassa aérea das árvores fertilizadas com lodo não diferiram das concentrações observadas no tratamento com fertilizante mineral; mesmo tendo o lodo adicionado ao solo cerca de 12 vezes mais $\mathrm{Zn}$ e $\mathrm{Cu}$. Isto pode estar relacionado à alta afinidade desses elementos pela matéria organica do solo, que porventura possa ter sido enrriquecida com a aplicação do lodo de esgoto, tornando estes elementos indisponível para absorção pelas plantas (DECHEN e NACHTIGALL, 2006)

No estoque de nutrientes foram observadas diferenças para todos os componentes, o que se deve à maior produção de biomassa obtida no tratamento com aplicação de lodo de esgoto (Tabelas 6 e 7). O componente que acumulou maior quantidade de nutriente em relação ao estoque total na parte aérea das árvores foi o lenho, variando aproximadamente de $35 \%$ (para o potássio) a $60 \%$ (para o fósforo). Por sua vez, a casca estocou de $10 \%$ (para o fósforo) a $30 \%$ (para o cálcio) do estoque total desses 
TABELA 4: Concentração dos macronutrientes $\left(\mathrm{g} \mathrm{kg}^{-1}\right)$ nos diferentes componentes da parte aérea do Corymbia citriodora, aos 4 anos de idade, adubado com lodo de esgoto e com fertilizante mineral.

TABLE 4: Macronutrients content $\left(\mathrm{g} \mathrm{kg}^{-1}\right)$ in different compartments of the Corymbia citriodora shoot, 4 years old, fertilized with sewage sludge and mineral fertilizer.

\begin{tabular}{|c|c|c|c|c|c|c|}
\hline Tratamento & $\mathrm{N}$ & $\mathrm{P}$ & $\mathrm{K}$ & $\mathrm{Ca}$ & $\mathrm{Mg}$ & $\mathrm{S}$ \\
\hline Lodo de esgoto & $2,1 \mathrm{~ns}$ & $0,29^{\mathrm{ns}}$ & 0,53 ns & $0,80^{\mathrm{ns}}$ & $0,10^{\mathrm{ns}}$ & $0,17^{\mathrm{ns}}$ \\
\hline Fertilizante mineral & 2,1 & 0,25 & 0,49 & 0,76 & 0,09 & 0,18 \\
\hline Lodo de esgoto & $3,3^{\mathrm{ns}}$ & $0,31^{\mathrm{ns}}$ & 2,0 ns & 3,5 ns & $0,75^{\mathrm{ns}}$ & $0,34^{\mathrm{ns}}$ \\
\hline Fertilizante mineral & 3,5 & 0,26 & 2,1 & 3,3 & 0,74 & 0,34 \\
\hline Lodo de esgoto & 4,8 ns & $0,53 \mathrm{~ns}$ & 2,4 ns & 2,3 ns & 0,54 ns & 0,38 ns \\
\hline Fertilizante mineral & 5,0 & 0,53 & 2,8 & 2,5 & 0,76 & 0,38 \\
\hline Lodo de esgoto & $17,4^{\mathrm{ns}}$ & $1,0 \mathrm{~ns}$ & $5,0^{\mathrm{ns}}$ & $2,2^{\mathrm{ns}}$ & 1,0 ns & $0,8^{\mathrm{ns}}$ \\
\hline Fertilizante mineral & 16,3 & 1,0 & 5,4 & 2,1 & 0,9 & 0,8 \\
\hline
\end{tabular}

Em que: * = Diferença significativa a $5 \%$ de probabilidade pelo teste $\mathrm{t}$; ${ }^{\mathrm{ns}}=$ Diferença não significativa pelo teste $\mathrm{t}$ $(\mathrm{P}>0,05)$.

TABELA 5: Concentração dos micronutrientes $\left(\mathrm{mg} \mathrm{kg}^{-1}\right)$ nos componentes da parte aérea do Corymbia citriodora, aos 4 anos de idade, adubado com lodo de esgoto e com fertilizante mineral.

TABLE 5: Micronutrients content $\left(\mathrm{mg} \mathrm{kg}^{-1}\right)$ in different compartments of the Corymbia citriodora shoot, 4 years old, fertilized with sewage sludge and mineral fertilizer.

\begin{tabular}{|c|c|c|c|c|c|}
\hline Tratamento & B & $\mathrm{Cu}$ & $\mathrm{Fe}$ & $\mathrm{Mn}$ & $\mathrm{Zn}$ \\
\hline$----\cdot$ & ----- & Lenh & ------- & ------- & ----- \\
\hline Lodo de esgoto & $4,4^{\mathrm{ns}}$ & $1,8^{\mathrm{ns}}$ & $43^{\text {ns }}$ & $34^{\text {ns }}$ & $16^{\text {ns }}$ \\
\hline Fertilizante mineral & 3,9 & 1,9 & 24 & 30 & 13 \\
\hline Lodo de esgoto & $9,6^{\mathrm{ns}}$ & $3,5^{\mathrm{ns}}$ & $87^{\mathrm{ns}}$ & $328^{\text {ns }}$ & $49^{\text {ns }}$ \\
\hline Fertilizante mineral & 8,5 & 3,8 & 76 & 340 & 44 \\
\hline Lodo de esgoto & $11,0^{\mathrm{ns}}$ & $11^{\text {ns }}$ & 67 ns & $243^{\text {ns }}$ & $54^{\text {ns }}$ \\
\hline Fertilizante mineral & 10,8 & 10 & 67 & 267 & 54 \\
\hline Lodo de esgoto & $16^{\mathrm{ns}}$ & $12^{\mathrm{ns}}$ & 164 ns & $218^{\mathrm{ns}}$ & $36^{\text {ns }}$ \\
\hline Fertilizante mineral & 19 & 11 & 150 & 201 & 40 \\
\hline
\end{tabular}

Em que: $*=$ Diferença significativa a $5 \%$ de probabilidade pelo teste $\mathrm{t} ;{ }^{\mathrm{ns}}=$ Diferença não significativa pelo teste $\mathrm{t}$ $(\mathrm{P}>0,05)$. 
nutrientes na biomassa aérea do Corymbia citriodora. $\mathrm{O}$ conhecimento da alocação de nutrientes nos componentes das plantas permite ao silvicultor optar por técnicas de colheita que reduzam a exportação de nutrientes do sítio (SANTANA et al., 2008).

$\mathrm{O}$ estoque de micronutrientes aos 4 anos de idade foi estatisticamente diferente entre os tratamentos estudados, mas os valores são pequenos e a colheita de toda a parte aérea não gera preocupação com o balanço desses elementos, pois as fertilizações efetuadas na rotina das empresas florestais adicionam quantidades suficientes de $\mathrm{B}, \mathrm{Cu}$ e $\mathrm{Zn}$ para a adequada nutrição das árvores; além disso, muitos solos com plantio de eucaliptos possuem quantidades consideráveis de Fe e Mn (SGARBY, 2002).

A quantificação das entradas e saídas de nutrientes (balanço de nutrientes) durante a rotação comercial do eucalipto é importante para orientar o processo de fertilização, que deve fornecer nutrientes para as plantas, assegurando o balanço dos nutrientes no ecossistema florestal (LACLAU et al., 2003).

Neste experimento verificou-se que o balanço nutricional (Tabela 8 ) foi negativo para o $\mathrm{N}$ e $\mathrm{K}$ em ambos os tratamentos, contudo, a quantidade de $\mathrm{N}$ que foi exportada com a colheita das árvores no tratamento com fertilização mineral $\left(-185 \mathrm{~kg} \mathrm{ha}^{-1}\right.$ de N) foi relativamente elevado, sendo superior à quantidade de $\mathrm{N}$ adicionada ao solo com a aplicação do fertilizante mineral no momento da implantação do experimento. Esse valor confirma a importância da devolução das folhas ao campo após a destilação do óleo essencial de Corymbia citriodora. Grande parte do produtores de óleo essencial devolvem em torno de $70 \%$ das folhas ao campo, sendo que os outros $30 \%$ são utilizados para abastecer as caldeiras da própria destilaria.

Ambos os tratamentos apresentaram balanço negativo para o potássio (Tabela 8), demonstrando haver a necessidade de adicionar maior quantidade de $\mathrm{KCl}$ ao solo nos próximos ciclos de produção. Apesar do lodo de esgoto possuir de forma geral baixa concentração de potássio em sua composição (Tabela 2), a matéria orgânica adicionada ao solo com a sua aplicação pode diminuir as perdas de $\mathrm{K}$ por lixiviação, principalmente devido ao aumento da capacidade de troca de catíons (CTC) do solo. Abreu Jr. et al. (2001) ao estudarem alguns atributos químicos em solos brasileiros fertilizados com composto de lixo urbano, verificaram que a aplicação isolada do composto de lixo em alguns solos ácidos promoveu a elevação da CTC de 7 \% a $117 \%$, e em solos alcalinos de $7 \%$ a $15 \%$.

TABELA 6: Estoque de macronutrientes $\left(\mathrm{kg} \mathrm{ha}^{-1}\right)$ nos diferentes componentes da parte aérea do Corymbia citriodora, aos 4 anos de idade, adubado com lodo de esgoto e com fertilizante mineral.

TABLE 6: Stock of macronutrients $\left(\mathrm{kg} \mathrm{ha}^{-1}\right)$ in the shoot components of Corymbia citriodora, 4 years old, fertilized with sewage sludge and mineral fertilizer.

\begin{tabular}{|c|c|c|c|c|c|c|}
\hline Tratamento & $\mathrm{N}$ & $\mathrm{P}$ & $\mathrm{K}$ & $\mathrm{Ca}$ & $\mathrm{Mg}$ & $\mathrm{S}$ \\
\hline \multicolumn{7}{|c|}{ 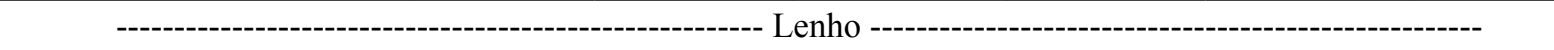 } \\
\hline Lodo de esgoto & $120,6^{\mathrm{A}}$ & $15,6^{\mathrm{A}}$ & $31,2^{\mathrm{A}}$ & $46,8^{\mathrm{A}}$ & $5,7^{\mathrm{A}}$ & $8,5^{\text {ns }}$ \\
\hline Fertilizante mineral & 94,5 & 12,4 & 23,6 & 34,9 & 4,5 & 7,8 \\
\hline Lodo de esgoto & $25,2^{\mathrm{A}}$ & $2,29^{\mathrm{A}}$ & $15,4^{\mathrm{A}}$ & $26,4^{\mathrm{A}}$ & $5,9^{\mathrm{A}}$ & $2,6^{\mathrm{A}}$ \\
\hline Fertilizante mineral & 21,4 & 1,55 & 12,9 & 20,3 & 4,5 & 2,1 \\
\hline Lodo de esgoto & $23,4^{\mathrm{A}}$ & $2,51^{\mathrm{A}}$ & $11,7^{\mathrm{A}}$ & $11,4^{\mathrm{A}}$ & $2,7^{\mathrm{ns}}$ & $1,9 *$ \\
\hline Fertilizante mineral & 18,6 & 1,96 & 10,4 & 9,5 & 2,7 & 1,5 \\
\hline Lodo de esgoto & $96,1^{\mathrm{A}}$ & $5,8^{\mathrm{A}}$ & $27,0^{\mathrm{A}}$ & $12,1^{\mathrm{A}}$ & $5,5^{\mathrm{A}}$ & $4,5^{\mathrm{A}}$ \\
\hline Fertilizante mineral & 73,1 & 4,5 & 20,2 & 9,4 & 4,1 & 3,6 \\
\hline & & & & & & \\
\hline Lodo de esgoto & $265,5^{\mathrm{A}}$ & $26,2^{\mathrm{A}}$ & $85,4^{\mathrm{A}}$ & $96,8^{\mathrm{A}}$ & $19,9^{\mathrm{A}}$ & $17,6^{\mathrm{A}}$ \\
\hline Fertilizante mineral & 207,7 & 20,3 & 67,2 & 74,1 & 15,8 & 15,1 \\
\hline
\end{tabular}

Em que: ${ }^{\mathrm{A}}=$ Diferença significativa a $5 \%$ de probabilidade pelo teste $\mathrm{t}$; ${ }^{\text {ns }}=$ Diferença não significativa pelo teste $\mathrm{t}$ $(\mathrm{P}>0,05)$. 
TABELA 7: Estoque de micronutrientes $\left(\mathrm{kg} \mathrm{ha}^{-1}\right)$ nos diferentes componentes da parte aérea do Corymbia citriodora, aos 4 anos de idade, adubado com lodo de esgoto e com fertilizante mineral.

TABLE 7: Stock of micronutrients $\left(\mathrm{kg} \mathrm{h}^{-1}\right)$ in the shoot components of Corymbia citriodora, 4 years old, fertilized with sewage sludge and mineral fertilizer.

\begin{tabular}{|c|c|c|c|c|c|}
\hline Tratamento & $\mathrm{B}$ & $\mathrm{Cu}$ & $\mathrm{Fe}$ & $\mathrm{Mn}$ & $\mathrm{Zn}$ \\
\hline \multicolumn{6}{|c|}{ 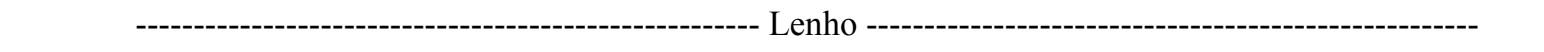 } \\
\hline Lodo de esgoto & $0,24 *$ & $0,10^{*}$ & $2,41 *$ & $1,94 *$ & $0,92 *$ \\
\hline Fertilizante mineral & 0,17 & 0,08 & 1,09 & 1,37 & 0,60 \\
\hline Lodo de esgoto & $0,07 *$ & $\begin{array}{l}- \text { Casca } \\
0,03 *\end{array}$ & $0,76^{*}$ & $2,49 *$ & $0,37 *$ \\
\hline Fertilizante mineral & 0,05 & 0,02 & 0,47 & 2,09 & 0,27 \\
\hline Lodo de esgoto & $0,05^{*}$ & $0,05^{*}$ & $0,32 *$ & $1,14^{*}$ & $0,26 *$ \\
\hline Fertilizante mineral & 0,04 & 0,04 & 0,25 & 0,79 & 0,20 \\
\hline & & - Folha & & & -- \\
\hline Lodo de esgoto & $0,09 *$ & $0,06^{*}$ & $0,91 *$ & $1,21^{*}$ & $0,20^{*}$ \\
\hline Fertilizante mineral & 0,08 & 0,05 & 0,67 & 0,90 & 0,18 \\
\hline Lodo de esgoto & $0,46^{*}$ & $\begin{array}{c}\text { - Total } \\
0,24^{*}\end{array}$ & $4,40^{*}$ & $6,78^{*}$ & $1,75^{*}$ \\
\hline Fertilizante mineral & 0,34 & 0,20 & 2,48 & 5,15 & 1,24 \\
\hline
\end{tabular}

Em que: $*$ = Diferença significativa a $5 \%$ de probabilidade pelo teste $\mathrm{t} ;{ }^{\mathrm{ns}}=$ Diferença não significativa pelo teste $\mathrm{t}$ $(\mathrm{P}>0,05)$.

TABELA 8: Balanço nutricional dos macronutrientes obtido com a exploração das folhas e da madeira nos tratamentos com aplicação de lodo de esgoto e de fertilizante mineral.

TABLE 8: Nutritional macronutrient balance with the harvest of leaves and wood, in treatments with application of sewage sludge and mineral fertilizer.

\begin{tabular}{|c|c|c|c|c|c|c|c|}
\hline \multirow{2}{*}{ Prática avaliada } & \multirow{2}{*}{ Tratamentos } & $\mathrm{N}$ & $\mathrm{P}$ & $\mathrm{K}$ & $\mathrm{Ca}$ & $\mathrm{Mg}$ & $\mathrm{S}$ \\
\hline & & \multicolumn{6}{|c|}{$\ldots . \mathrm{kg} \mathrm{ha}^{-1} \ldots}$. \\
\hline \multirow{2}{*}{ Colheita das folhas até o $3^{\circ}$ ano } & Lodo & 100 & 5 & 32 & 20 & 7 & 3,3 \\
\hline & Mineral & 92 & 5 & 30 & 18 & 6 & 3,1 \\
\hline \multirow{2}{*}{$\begin{array}{c}\text { Colheita aos } 4 \text { anos } \\
\text { (todos os componentes aéreos) }\end{array}$} & Lodo & 265 & 26 & 85 & 97 & 20 & 18 \\
\hline & Mineral & 208 & 20 & 67 & 74 & 16 & 15 \\
\hline \multirow{2}{*}{ Total das colheitas } & Lodo & 365 & 31 & 117 & 117 & 27 & 21 \\
\hline & Mineral & 300 & 25 & 97 & 92 & 22 & 18 \\
\hline \multirow{2}{*}{ Aplicado via adubação } & Lodo & 320 & 144 & 102 & 248 & 48 & 66 \\
\hline & Mineral & 115 & 60 & 80 & 440 & 160 & 5 \\
\hline \multirow{2}{*}{ Balanço (aplicado - exportado) } & Lodo & -45 & 113 & -15 & 131 & 21 & 45 \\
\hline & Mineral & -185 & 35 & -17 & 348 & 138 & -13 \\
\hline
\end{tabular}


O tratamento com fertilizante mineral apresentou balanço negativo também para $\mathrm{o}$ enxofre, mesmo tendo sido exportada uma pequena quantidade de $\mathrm{S}$ relativa à quantidade dos demais nutrientes exportados; todavia, isto não deve ocasionar problemas para os ciclos de produção futuros, visto que no Brasil a deficiência de $\mathrm{S}$ em plantios florestais é raramente constatada (SILVEIRA et al., 2005).

Em contrapartida, ambos os tratamentos apresentaram balanço positivo para os nutrientes $\mathrm{P}, \mathrm{Ca}$ e $\mathrm{Mg}$. No tratamento com lodo de esgoto, a quantidade de fósforo excedente após todas as exportações com a colheita das folhas e da madeira possibilitaria implementar até três novos ciclos de produção, desde que não fossem contabilizados outros tipos de perdas ou de indisponibilização do nutriente para a absorção pelas plantas (por exemplo a adsorção de $\mathrm{P}$ em óxidos de ferro e alumínio). Sob a mesma perspectiva, no tratamento com fertilização mineral, as quantidades de cálcio e magnésio excedentes possibilitariam que fossem conduzidos respectivamente de 3 a 6 novos ciclos de produção. Segundo McLaren et al. (2007) os efeitos em longo prazo (residual) dos nutrientes adicionados ao solo devido à aplicação do lodo de esgoto são ainda objetos de muita discussão e de considerável interesse pela comunidade científica mundial.

\section{CONCLUSÕES}

A aplicação de lodo de esgoto complementado com potássio e boro resulta em maior produtividade de biomassa foliar, óleo essencial e de madeira do Corymbia citriodora. Com isso, a exportação de nutrientes com a colheita das folhas e da madeira é maior no plantio fertilizado com lodo de esgoto comparado ao cultivo com fertilizante exclusivamente mineral.

A fertilização do plantio de Corymbia citriodora com lodo de esgoto proporciona maior equilíbrio entre a entrada e a saída (balanço) de nutrientes do ecossistema, principalmente no caso do nitrogênio, se comparado ao plantio com fertilização mineral.

\section{REFERÊNCIAS BIBLIOGRÁFICAS}

ABREU JUNIOR, C. H. et al. Cátions trocáveis, capacidade de troca de cátions e saturação por bases em solos brasileiros adubados com composto de lixo urbano. Scientia Agrícola, Piracicaba, v. 58, n. 4, p. 813-824, out./dez. 2001.

ANDRADE, G. C. et al. Acúmulo de nutrientes na biomassa e na serapilheira de Eucalyptusgrandis em função da aplicação de lixo urbano e de nutrientes minerais. Boletim de Pesquisa Florestal, Colombo, n. 53, p. 109-136, jul./dez. 2006.

BATISH, D. R. et al. Chemical composition and phytotoxicity of volatile essential oil from intact and fallen leaves of Eucalyptus citriodora. ZeitschriftfurNaturforschung - Section C,Biosciences, v. 61, n. 7/8, p. 465-471, 2006.

BELlOTE, A. F. J.; SILVA, H. D. Técnicas de amostragem e avaliações nutricionais em plantios de Eucalyptus spp. In: GONGALVES, J. L. M.; BENEDETTI, V. (Org.). Nutrição e Fertilização Florestal. Piracicaba: IPEF, 2005. p. 105-133.

CAMARGO, O. A. et al. Método de análise química, mineralógica e física de solos do Instituto Agronômico de Campinas.Campinas: Instituto Agronômico, 1986. 94 p. (IAC, Boletim Técnico, 106).

CETESB - Companhia de Tecnologia de Saneamento Ambiental. Norma P 4.230: Aplicação de lodos de sistemas de tratamento biológico em áreas agrícolas - Critérios para projeto e operação. São Paulo, 1999. 32 p.

CONAMA - Conselho Nacional do Meio Ambiente. Resolução No. 375. Define critérios e procedimentos, para o uso agrícola de lodos de esgoto gerados em estações de tratamento de esgoto sanitário e seus produtos derivados, e dá outras providências. Ministério do Meio Ambiente, 2006.

DECHEN, A. R.; NACHTIGALL, G. R. XIII Micronutrientes. In: FERNANDES, M. S. (Edit.). Nutrição Mineral de Plantas. Viçosa, MG: Sociedade Brasileira de Ciência do Solo, 2006. p. 327-354.

EMBRAPA. Centro Nacional de Pesquisa de Solos. Sistema brasileiro de classificação de solos. 2 . ed. Rio de Janeiro, 2006. 306 p.

HARRISON, R. B. et al. Reciclagem de resíduos industriais e municipais em áreas de reflorestamento. Circular Técnica (IPEF), Piracicaba, n. 198, p. 1-21, jul. 2003.

LACLAU, J. P. et al. Nutrient dynamics throughout the rotation of Eucalyptus clonal stands in Congo.Annals of Botany, England, v. 91, n. 7, p. 879-892, Jun. 2003.

LACLAU, J. P. et al. The function of the superficial root mat in the biogeochemical cycles of nutrients 
in Congolese Eucalyptus plantations.Annals of Botany, England, v. 93, n. 3, p. 249-61, Mar. 2004. MALAVOLTA, E. et al. Avaliação do estado nutricional das plantas: Princípios e aplicações. Piracicaba: POTAFOS, 1997. 319 p.

McLAREN, R. G. et al.Distribution andmovement of nutrients and metals in a Pinusradiata forest soil following applications of biosolids. Environmental pollution, New Zealand, v. 147, p. 32-40, May. 2007.

PEREIRA, A. R. et al. Produção de biomassa e remoção de nutrientes em povoamentos de Eucalyptuscitriodora e Eucalyptussaligna cultivados na região de cerrado de Minas gerais. Floresta, Curitiba. v. 15, n. 1/2, p. 8-16, 1984.

POGGIANI, F. et al. Ciclagem e exportação de nutrientes em florestas para fins energéticos. IPEF, n.27, p.17-30, ago. 1984.

POGGIANI, F. et al. Uso do lodo de esgoto em plantações florestais. In: PROSAB - Programa de Pesquisa em Saneamento Básico. In: ANDREOLI, C. V. Biossólidos -Alternativas do Uso de Resíduos do Saneamento. Rio de Janeiro: ABES RJ, 2006, v. 4, p. 159-188.

RAIJ, B. van. et al. Análise química do solo para fins de fertilidade.Campinas: Fundação Cargill, 1987. $170 \mathrm{p}$.

RAIJ, B. van. et al. Recomendações de adubação e calagem para o estado de São Paulo. Campinas: Instituto Agronômico/Fundação IAC, 1996, 285 p. (IAC. Boletim Técnico 100).

ROLIM, G. S.; SENTELHAS, P. C. Balanço hídrico normal por Thorntwaite e Mather (1955). Piracicaba: ESALQ/USP - Departamento de Ciências Exatas: Área de Física e Meteorologia, 1999 (programa para Excel v. 6).
SANTANA, R. C. et al. Biomassa e conteúdo de procedências de Eucalyptusgrandis e Eucalyptussaligna em alguns sítios florestais do estado de São Paulo. Scientia Forestalis, Piracicaba, n. 56, p. 155-169, dez. 1999.

SANTANA, R. C. et al. Estimativa de biomassa de plantios de eucalipto no Brasil. Revista Árvore, Viçosa, v. 32, n. 4, p. 697-706, jul./ago. 2008.

SAS INSTITUTE. SAS user's guide: statistics. Cary, 2002-2003.

SGARBY, F. Produtividade de Eucalyptussp. em função do estado nutricional e da fertilidade do solo em diferentes regiões do estado de São Paulo, 2002. 101 f. Dissertação (Mestrado em Recursos Florestais) - Escola Superior de Agricultura "Luiz de Queiroz", Universidade de São Paulo, Piracicaba, 2002.

SILVA, P. H. M. et al. Crescimento de Eucalyptusgrandis tratado com diferentes doses de lodos de esgoto úmido e seco, condicionados com polímeros. Scientia Forestalis, Piracicaba, v.36, n. 77, p. 79-88, mar. 2008.

SILVA, P. H. M. et al. Produção de óleo essencial e balanço nutricional em Corymbiacitriodora adubado com lodo de esgoto em diferentes espaçamentos Cerne, Lavras,v. 15, n. 3, p. 346-354, jul./set. 2009. SILVEIRA, R. L. V. et al. Avaliação do estado nutricional do Eucalyptus: Diagnose visual, foliar e suas interpretações. In: GONGALVES, J. L. de M.; BENEDETTI, V. (Org.). Nutrição e Fertilização Florestal. Piracicaba, SP: IPEF, 2005. p. 79-104. ZAIA, F. C.; GAMA-RODRIGUES, A. C. Ciclagem e balanço de nutrientes em povoamentos de eucaliptos na região norte fluminense. Revista Brasileira de Ciência do Solo, Viçosa, v. 28, n. 5, p. 843-852, set./out. 2004. 